\title{
Managing Dynamic Context to Optimize Smart Interactions and Services
}

\author{
Norha M. Villegas ${ }^{1,2}$ and Hausi A. Müller ${ }^{1}$ \\ ${ }^{1}$ University of Victoria \\ hausi@cs.uvic.ca \\ ${ }^{2}$ Icesi University \\ nvillega@cs.uvic.ca
}

\begin{abstract}
With the rapid growth of socio-technical ecosystems, smart interactions and services are permeating every walk of life. As smart interactions must managed automatically and interactively in response to evolving user's matters of concern, the smart Internet requires creative approaches where services and interactions are implemented with awareness of, and dynamic adaptation to, users, computational environments, changing policies and unknown requirements. Consequently, modeling and managing dynamic context is critical for implementing smart services and smart interactions effectively. Thus, smart interactions need infrastructure to acquire, compose, and distribute context information to multiple execution endpoints. Moreover, context management must be controlled and governed to optimize system properties. This chapter surveys context modeling and management approaches intended for the optimization of smart interactions and services, discusses the main challenges and requirements of context-awareness in the smart Internet, and provides a feature-based framework useful for the evaluation and implementation of context modeling and management mechanisms.
\end{abstract}

Keywords: dynamic context, context-awareness, context modeling, dynamic context management, feature-based context characterization survey, feedback control, smart interactions, smart services, smart Internet.

\section{Introduction}

Nowadays, systems are evolving from software intensive systems to socio-technical ecosystems, where dynamic groups of users, stakeholders, businesses, and software and hardware infrastructures, have to cooperate in complex and changing environments [1. The World Wide Web is a good representative of such systems. Generally, its stakeholders have uncertain, conflicting, and changing requirements; furthermore, its control is highly complex and decentralized, and its users project an increasing need for dynamic and personalized services.

In this endeavour of providing dynamic applications centered on the user's goals, several researchers have made important contributions. One such initiative is the notion of Smart Internet introduced at the Smart Internet Technologies Working 
Conference (SITCON) at CASCON 2009 1 The Smart Internet includes two specific areas of research. Smart Interactions, which deal with aspects related to the discovery, aggregation, and delivery of resources from the Internet (e.g., the automatic deployment of components to provide personalized services to a persontraveler - who plans a trip involving transportation, accommodation, dining, and shopping); and Smart Services, which focus on providing the suitable infrastructure to support smart interactions (e.g., the required functionality to support the dynamic adaptation of the system, based on the traveler's environment and preferences) [2. In order to support smart interactions and smart services, a system should be able to reason about its current state and provide functionality based on its context and the current user's matters of concern (mocs). In other words, such a system has to be reflective and context-aware. Thus, dynamic, user-centric applications require context management infrastructures able to provision context information by implementing self-managing interactions and services for supporting policy-based composition of context facts. We advocate creative approaches not just to acquire, model and distribute context information to multiple endpoints, but also to control and govern its provisioning, maintenance, and evolution 3 .

This chapter reports on the results of a survey of context modeling and context management approaches in different problem domains. This study was performed with the motivation of identifying context modeling and management requirements for supporting smart interactions and services, and guided by four main research questions related to the challenges of context-awareness in the light of the smart Internet: (i) How to identify relevant context and the corresponding context management objectives for a particular set of interactions involved in a $m o c$ ? (ii) How to acquire, compose, and distribute context information to multiple execution endpoints in an efficient manner? (iii) How to manage the dynamic nature of context information and the uncertainty of the context-aware system's requirements? (iv) How to control and govern context management by ensuring its provisioning according to the user's mocs and system's requirements?

To conduct this exploration, we propose an operational definition of context derived from three important existing definitions. The first one is the classic definition proposed by Dey et al. 4. The second one is the operational extension of this definition given by Zimmermann et al. [5]. Our operational definition is a combination of these two definitions and the notion of context life cycle applied by Hynes [6]. An operational definition has the advantage of being more concrete and directly implementable than just abstract/conceptual definitions. Furthermore, we propose a context taxonomy based on previous classifications of context information. According to our taxonomy, context information can be classified into five fundamental categories: individuality, time, location, activity and relational (i.e., social, functional and compositional relationships among the first four types). To conduct our survey, we applied the method of FeatureOriented Domain Analysis (FODA) proposed by researchers of Carnegie Mellon Software Engineering Institute (CMU SEI) [7]. The main focus of this method is the identification of prominent or distinctive features of software systems in a

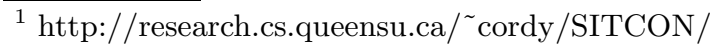

